\section{Cahiers de Narratologie}

Analyse et théorie narratives

33 | 2018

L'Art du roman chez Umberto Eco

\title{
Une rose sans épines : apostille à l'« Apostille au Nom de la rose »
}

\section{Massimo Leone}

\section{(2) OpenEdition}

\section{Journals}

\section{Electronic version}

URL: http://journals.openedition.org/narratologie/8039

DOI: 10.4000/narratologie.8039

ISSN: $1765-307 X$

Publisher

LIRCES

\section{Electronic reference}

Massimo Leone, «Une rose sans épines : apostille à l'« Apostille au Nom de la rose » », Cahiers de Narratologie [Online], 33 | 2018, Online since 23 July 2018, connection on 19 April 2019. URL : http:// journals.openedition.org/narratologie/8039; DOI : 10.4000/narratologie.8039

This text was automatically generated on 19 April 2019

Article L.111-1 du Code de la propriété intellectuelle. 


\title{
Une rose sans épines : apostille à l'« Apostille au Nom de la rose»
}

\author{
Massimo Leone
}

«Ma neppure io la so, questa maledetta

natatione!»

« Impara. »

Umberto Eco, L’isola del giorno prima (1994).

\section{Introduction : narration et théorisation}

1 La sémiotique d'Umberto Eco se prolonge par d'autres moyens dans ses romans : on a beaucoup écrit à ce sujet. Mais l'inverse est vrai aussi : les romans d'Umberto Eco se propagent par des voies sibyllines dans son œuvre philosophique. La prodigieuse capacité de raconter du grand savant italien se manifeste bien avant le Nom de la rose et s'applique principalement aux idées et au savoir. Chez Eco, le roman ne fait qu'habiller par des acteurs, des figures, des icônes, ainsi que par un agencement génial de lieux, de temps, et d'aspects, une structure narrative très solide, héritière des grandes narrations populaires du dix-neuvième siècle.

2 Mais ce n'est pas que dans les romans d'Eco que l'on retrouve Dumas : il suffit de lire une page, parfois même une phrase des essais du sémioticien pour y reconnaître immédiatement l'auteur. Cette reconnaissance foudroyante, qui fait également le grand succès éditorial d'Umberto Eco, se doit tantôt à la formulation d'idées perspicaces, tantôt à la verve rhétorique les exprimant, mais elle est due surtout à l'affabulation narrative de l'auteur. Depuis toujours, peut-être même à partir de la thèse qu'Umberto Eco écrivit, jeune philosophe, pour obtenir sa laurea auprès de l'Université de Turin, la prose académique qu'il déploie met en scène la bataille des idées, un combat abstrait dont on suit néanmoins l'évolution et les coups de théâtre comme s'il s'agissait des protagonistes d'un roman.

3 Cela se passe, chez Eco, non pas dans la forme assez banale de la prosopopée académique, où des concepts et des théories opposées s'affronteraient derrière les noms de grands 
savants, mais par le biais d'une stratégie rhétorique plus subtile, que l'on dirait même maïeutique: dans Le nom de la rose, le lecteur modèle est invité à découvrir la vérité derrière le mystère en suivant les abductions hardies de Guillaume da Baskerville ; dans les essais d'Eco, le lecteur modèle est encouragé à endosser le rôle de héros du savoir. Eco et ses interprètes ont souvent insisté sur le fait qu'il concevait un roman comme une écriture destinée à raconter ce qu'on ne peut pas expliquer; mais là aussi se cache le soupçon d'une symétrie que les exégètes ont souvent négligée : chez Eco, le pouvoir de la narration ne se manifeste pas uniquement lorsque l'explication échoue mais également lorsqu'elle triomphe.

4 En d'autres mots, les romans ne font que conduire aux conséquences ultimes une attitude de pensée, de composition et de style qui domine toute la prose du polymathe italien. Mais qu'est que cela signifie, que la narration peut dépasser l'explication? Et dans quel sens la prose académique d'Umberto Eco exploite-t-elle ce pouvoir?

5 On s'est souvent abreuvé à "l'Apostille au Nom de la rose » (1983), l'essai qu'Eco publia dans la revue Alfabeta afin de rendre compte de son imagination et de son écriture narrative, pour tâcher de dévoiler les secrets de son attrait ainsi que de son succès planétaire. En effet, dans cet essai, le narrateur Eco s'explique et, en partie, se dévoile, mais, comme toujours chez lui, cette révélation se déploie sur plusieurs couches, les plus profondes pouvant être atteintes uniquement par ceux qui lisent la prose d'Eco non seulement dans son contenu, mais également dans sa forme. Pour qui lit, relit, voire mémorise l'œuvre sémiotique d'Umberto Eco, un élément singulier retient son attention : on pourrait le dénommer l'alternance entre la rigueur et l'impertinence. Dans «l'Apostille », par exemple, l'auteur insiste beaucoup sur des ingrédients de la narration qui, reconnectés dans l'isotopie de la lecture, renvoient tous à l'image d'un écrivain essentiellement artisan, constructeur, calculateur, mais surtout à l'image d'une rationalité combinatoire primant sur toute dynamique irrationnelle de la fantaisie: l'inspiration, l'intuition, le génie.

6 Lorsqu'il explique l'origine du titre quelque peu mystérieux de son roman best-seller, Eco répète ce qu'il a argumenté maintes fois dans ses écrits académiques : le roman est une «machine pour générer des interprétations ». Ceux qui lisent cette phrase dans les essais d'Eco auront l'impression de faire face à une posture intellectuelle foncièrement rationaliste, laquelle rejette toute évocation romantique, idéaliste, sentimentale de la littérature pour la transformer en fait de langage, explicable dans un cadre théorique étant, à l'origine, celui du structuralisme saussurien. De manière très sophistiquée, dans la sémiotique d'Eco, l'expulsion de la subjectivité créatrice qui est insaisissable hors de la langue - expulsion qui démarre avec Saussure et théoriquement s'achève avec Louis Trolle Hjelmslev - s'applique à la culture dans son ensemble.

7 Umberto Eco domine toute l'histoire intellectuelle italienne de la deuxième moitié du vingtième siècle, mais cette suprématie, comme toutes les suprématies, se construit stratégiquement par la démolition - encore plus efficace car implicite - de la pensée de celui qui avait dominé la culture italienne pendant la première moitié du vingtième siècle, à savoir Benedetto Croce. Cela serait exagéré d'affirmer que l'idéologie sémiotique d'Eco, c'est-à-dire sa façon d'imaginer la nature du sens et du langage, y compris le sens littéraire, est spéculaire par rapport à l'idéologie sémiotique de Croce. Cependant, à chaque fois qu'Eco cite Benedetto Croce dans ses essais, par exemple dans le Trattato di semiotica generale (1975), l'on est souvent sûr de se trouver à un tournant essentiel de l'argumentation, car en définitive toute la pensée du sémioticien, son succès académique, 
mais également sa mission intellectuelle pourraient se résumer dans la nécessité de rebâtir la culture italienne dans son ensemble, en n'en choisissant pas comme pilier l'idée de la force de l'esprit - tentation constante dans l'histoire culturelle italienne -, mais en désagrégeant ce concept sous les coups d'une analyse impitoyable, montrant à chaque pas que des structures culturelles essentiellement sociales se cachent derrière toute proclamation de génie.

Cette mission, on le comprend bien, n'est pas uniquement théorique. L'Italie qui cède au désir de l'individualité inspirée se hissant au-dessus de l'histoire par ses propres forces coïncide souvent avec l'Italie qui s'abandonne à la pensée du chef tout-puissant, de l'homme qui, doué, ou plutôt surdoué de qualités exceptionnelles, forge son destin en même temps que le destin de la "patrie ». D'ici découle l'urgence, lorsque le même Umberto Eco devient l'objet d'une attribution généralisée de génie (notamment après le succès extraordinaire du Nom de la rose), de la démanteler, ou du moins de montrer que ce génie, lui aussi, ne dérive pas d'une potion mystérieuse que l'auteur et peu d'élus auraient à leur portée, mais que ce génie jaillit, au contraire, essentiellement d'un jeu tout à fait rationnel avec la langue et dans le langage.

\section{Le titre et le sens}

Pourquoi Eco a-t-il choisi ce titre foudroyant de Le nom de la rose? Car il aurait été visité, soudainement, par une vision mystique? Pas du tout! Dans l'Apostille, Eco frustre cette idée en la banalisant et surtout en la rationalisant : le titre lui est venu à l'esprit « presque par hasard "; en plus, comme la rose est "une figure symbolique ainsi chargée de significations qu'elle n'en a presque plus aucune », elle était parfaite pour "égarer le lecteur ». Mais cela serait très ingénu de croire que, suite à cette stratégie de banalisation du romantisme de la création littéraire, les lecteurs en seraient convaincus et, ce qui est plus important, désenchantés. Au contraire, face à la posture intellectuelle de celui que l'on croit dépositaire de génie, et qui, de façon paradoxale, vous explique que ce génie est à la portée de tout le monde, l'admiration ne décroit pas du tout, elle augmente, car l'on est devant quelqu'un qui non seulement étonne par la parole, mais qui, par une métaparole, dévoile les sources publiques de cet étonnement. Eco ne se veut pas tant un magicien de la prose qu'un prestidigitateur : ses ruses laissent abasourdi, mais à aucun moment il ne vous fait croire qu'elles ne sont pas le fruit d'une technique. La vénération qui s'en suit se retrouve donc doublée : car on admire non pas seulement l'effet de magie, mais aussi la magie de l'effet.

\section{Raconter le procès}

"Les écrits de poétique », affirme Eco dans "l'Apostille ", "ont la fonction d'aider à comprendre comment se résout ce problème technique qui est la production d'une œuvre »; « problème ", « technique », « production », « œuvre » : ces termes, tout comme la définition de roman en tant que "machine " qui les précède dans le même essai, et comme beaucoup d'autres termes et définitions qui lui suivront, sont on ne peut plus "spoetizzanti », un terme italien qui n'a pas d'égal en français mais qui littéralement signifie « qui ôte de la poésie aux choses ", qui dépoétise. Le roman est une "machine »; sa création est un "problème technique de production »: on dirait que ce n'est pas un écrivain qui parle, mais un ingénieur. D'une part, l'on est tenté de lui attribuer la tonalité 
glaciale de l'explication structuraliste ; d'autre part, on y renifle, surtout lorsque le roman devient une œuvre que l'on "produit», une idéologie marxiste de la culture, qui est pensée en termes de « moyens de production».

Les deux courants de pensée s'entrecroisent chez Eco mais il serait hâtif d'attribuer sa vision machinale du roman à l'un ou à l'autre. Le sémioticien continue dans le même essai : «Celui qui écrit (ou peint ou sculpte ou compose de la musique) sait toujours ce qu'il fait et combien cela lui coûte. Il sait qu'il doit résoudre un problème. Il se peut que les données de départ soient obscures, pulsionnelles, obsessionnelles, pas plus qu'une envie ou un souvenir. Mais après, le problème se résout « a tavolino ». Encore une image "spoetizzante »: résoudre un problème "a tavolino " évoque, en italien, l'image d'un individu ou d'un groupe d'individus assis autour d'une " petite table "; l'expression qui lui est la plus proche, en français, serait « décider de quelque chose sur le papier »; mais «a tavolino » est une image encore moins poétique, on ne lui reconnaît même pas la dignité littéraire du papier ; c'est une image de petite bourgeoisie se retrouvant autour d'un mobilier sans chichis pour y faire un quelconque bilan familier.

D'un côté, l'attitude d'Eco est quelque peu gênante : le lecteur veut croire au mythe de l'inspiration du poète, de l'écrivain, de l'artiste; il veut se l'imaginer lorsque le vent d'une puissance supérieure l'emporte vers des hauteurs inaccessibles aux mortels; mais l'artiste aussi veut y croire : pourquoi réduire sa création à un exercice de comptabilité ? Ce serait comme dire à quelqu'un qui est amoureux que ce sont, en effet, seulement les hormones qui le mettent dans cet état. L'effet de banalisation est ultérieurement renchéri par le dédain qu'Eco montre vis-à-vis tout ce qui précède l'ingénierie de la création littéraire : «les données de départ », « obscures, pulsionnelles, obsessionnelles ». Mais ce n'est pas exactement cela qui, dans la littérature, nous intéresse? Ce n'est pas exactement ces sujets que nous interrogeons, depuis toujours?

\section{Naturellement, le Moyen Âge}

Dans « l'Apostille », Eco ne se dévoile point. «J'ai écrit un roman parce que l'envie m'en en est venue ", écrit-il. "Je crois - il ajoute - que cela est une raison suffisante pour se mettre à raconter "; "l'homme est un animal fabulateur par nature », conclue-t-il. Mais l'est-il vraiment? Peut-être dans le sens très abstrait de l'herméneutique de Paul Ricœur, ou de la sémiotique générative d'Algirdas J. Greimas, à savoir dans le sens où l'homme structure l'intelligibilité de la réalité par la narration, ou mieux, par la narrativité. Mais est-ce l'homme naturaliter fabulateur dans le sens plus spécifique qu'il ou elle est naturellement induit(e) à raconter des histoires, comme Eco le fait de façon excellente à la fois dans ses romans et dans ses essais ? L'évidence empirique dément cette hypothèse : la plupart des essais académiques sont ennuyeux - ou, du moins, n'amusent pas comme les essais d'Umberto Eco - car ils sont tout à fait incapables de produire les trois effets pragmatiques que le sémioticien italien maitrisait parfaitement : raconter, surprendre, amuser. Plusieurs des grands philosophes de la deuxième moitié du vingtième siècle, par contre, ont atteint un public considérable par un discours très abstrait, qui ne se faisait ni récit, ni coup de théâtre, ni blague. Non, lorsqu'Eco explique l'origine du roman en disant que « l'homme est fabulateur par nature » il joue encore une fois à cache-cache, essayant de détourner l'attention du lecteur de «l'Apostille» de la seule position empiriquement acceptable: c'est Eco qui est un fabulateur par nature, mais il ne veut pas expliquer pourquoi. 
Eco ajoute ensuite: "J'ai commencé à écrire en mars 78, mu par une idée séminale ; j'avais envie d'empoisonner un moine "; c'est un chef-d'œuvre de réponse car, au lieu de fournir des éléments pour expliquer la genèse du roman en relation à la biographie psychologique de l'auteur (j'ai commencé à écrire après la mort de mon père ; après que je me suis décidé d'abandonner la religion catholique; après une grande déception amoureuse; etc.), il l'explique en relation avec le noyau narratif central du roman, à savoir il ne l'explique pas du tout. Qu'est-ce que cela veut dire, d'écrire un long roman parce que l'on a envie « d'empoisonner un moine » ? Les psychanalystes s'amuseront dans la tentative de déchiffrer cet aveu, mais le métier du sémioticien est autre : souligner que, lorsqu'il écrit un essai censé dévoiler certains aspects de l'écriture du Nom de la rose, Eco finit par raconter sans se raconter, composant, ainsi, un méta-roman ayant pour objet l'écriture du roman même.

15 Ce qui en dérive est une sorte de structure fractale où, par exemple, Eco révèle, peu après, avoir retrouvé un cahier daté 1975 où il avait «dressé une liste de moines dans un monastère inconnu»; ce qui ne fait que reproduire, dans le discours de l'Apostille, le même stratagème - celui de la mention d'un manuscrit retrouvé - par lequel s'ouvre Le nom de la rose, ainsi que d'autres romans historiques. Ceux qui croient à cette révélation ne s'aperçoivent pas qu'Eco y glisse un nouveau divertissement, dont le but est, encore une fois, de détourner l'attention du lecteur de l'idée d'inspiration, de construire une source imaginaire - quoiqu'il s'agisse d'une sorte d'auto-source-pour la création littéraire, de laisser le lecteur tout seul, derechef, avec ses inférences. Eco a retrouvé une liste de moines dressée en 1975 ; mais pourquoi l'a-t-il dressée en première instance? Qu'est-ce qui l'a poussé à agir ainsi ? Et à cette révélation Eco ajoute : « rien d'autre ». La vigueur de la réticence consiste à créer de la tension narrative - la même que l'auteur distille de façon magistrale dans le récit du Nom de la rose - par la construction d'un secret. Le frisson qu'un secret nous procure - les rhétoriciens le savent très bien - ne dépend pas de la nature de ce que l'on cache, mais de la nature de ce avec quoi on le cache. Si Eco nous avait dit: le moine représente pour moi l'éducation religieuse que, enfant, j'ai reçue de mon père ; en le tuant, j'ai tué symboliquement mon père ; cela serait une révélation de quelque chose de secret mais il s'agirait d'une révélation kitsch, presque ridicule; au contraire, Eco nous raconte d'un manuscrit inconnu, il ajoute ce «nulla d'altro », " rien d'autre », banalise, ainsi, l'étiologie psychologique de son roman mais, ce faisant, il construit un méta-secret (un secret sur la genèse d'un roman traitant d'un secret), ce qui alimente encore plus la curiosité du lecteur. D'où vraiment a-t-il pris l'idée du Nom de la rose? On est donc presque forcé de continuer la lecture de «l'Apostille » pour le savoir.

Par la dénégation du rôle de l'inspiration d'une part, et par l'occultation de la source de cette inspiration de l'autre, Eco donne parfois même l'impression que son roman s'est écrit par lui-même, qu'il n'a fait que prêter sa (prodigieuse) capacité combinatoire à un récit qui était déjà présent dans la grammaire narrative de la culture. Parfois, cette technique d'affabulation sur l'affabulation est si évidente qu'elle résulte même quelque peu ornementale, par exemple lorsqu'Eco raconte, toujours dans la suite de "l'Apostille », qu'il s'est fait prescrire, par un ami biologiste, un poison en tout point semblable à celui du Nom de la rose, et que lui, Eco, avait ensuite détruit la lettre du biologiste car il (le sémioticien) craignait que ce document "pût être lu dans un autre contexte ». C'est de l'affabulation pure. Un romancier n'a nul besoin de ce genre de conseils, car c'est le 
lecteur du roman qui en a besoin : lorsque le roman lui dit qu'il y a une substance $\mathrm{X}$ qui tue de la façon $Y$, il y croit.

$\mathrm{Au}$ contraire, ce récit sur le récit contribue à créer la mythologie publique de l'auteur Eco, et faire imaginer que, pendant la gestation de ses romans, il entretient une correspondance avec toute sorte de savants afin de vérifier les détails de la narration. Cela est peut-être vrai (de moins en moins, probablement, pour les romans d'Eco écrits après l'invention d'Internet), mais, de nouveau, l'auteur ne nous dit rien sur l'origine du roman en tant qu'expression d'une subjectivité. Nous avons le texte d'une part, expression combinatoire des possibilités d'un système de signes très complexe, et le mythe de l'auteur de l'autre. Eco savait très bien que l'écrivain contemporain doit non seulement raconter mais se raconter, se mettre en scène en tant qu'écrivain, une tendance qui séduit les masses et qui, avec l'explosion des réseaux sociaux, devient presque caricaturale : plusieurs jeunes écrivains contemporains passent moins de temps à écrire qu'à soigner leur image dans les réseaux sociaux, en y postant toutes les photos de tous les lecteurs qui lisent leurs livres, par exemple.

La place qu'Eco laisse vide, au milieu, entre un texte impersonnel et le simulacre médiatique de l'écrivain, est celle de la personnalité de l'auteur, de sa sensibilité, de ses craintes et de ses chagrins, des sources intimes, et donc ultimes, de sa nécessité de raconter. Dans une illusion admirable, Eco nous propose les effets comme causes, et donc il continue à cacher les causes en créant autour d'elles une auréole subreptice de mystère; il est évident que dresser une liste de moines, vouloir en empoisonner un, chercher le poison parfait pour le faire, ne sont pas la cause de la narration mais les effets de son désir. Cependant, c'est en tant que causes qu'Eco nous les présente.

De même, lorsqu'il s'interroge et répond sur les raisons qui l'ont poussé à choisir le Moyen Âge, Eco ne se découvre jamais : il nous fait savoir que, pour écrire son roman, il a puisé dans les dossiers qu'il avait accumulés pour ses travaux académiques, mais il ne touche pas à la question fondamentale : pourquoi le Moyen Âge? Qu'y a-t-il, dans cette époque, qui a sollicité l'esprit de l'écrivain et son imagination narrative? Là encore, rien ne nous est dévoilé à ce sujet. Eco arrive même à raconter un épisode assez personnel : son épouse lui reprochait de ne pas savoir regarder les étincelles qui se levaient d'un feu de camp, étincelles qui apparurent ensuite dans Le nom de la rose ; mais la conclusion d'Eco est qu'il "savait les regarder avec les yeux d'un homme médiéval »; là aussi, dans l'évocation de l'intimité du couple, l'homme Eco disparait pour ne réapparaitre que comme mémoire et écriture impersonnelle, presque sacerdotale, du savoir.

\section{Le masque}

20 Il est assez significatif que, dans «l'Apostille », le seul sentiment personnel qu'Eco avoue est la honte: "J'avais honte à raconter", une affirmation qui, en quelque sorte, jure à côté de celle où Eco nous disait que «l'homme est fabulateur par nature ». En effet, là aussi, l'homme-écrivain se cache derrière l'écrivain-druide de la culture : ce n'est point Eco qui parle mais les chroniqueurs médiévaux ou, mieux encore, c'est le réseau intertextuel qui parle, d'une façon qui, encore une fois, limite l'intervention personnelle de l'auteur à un choix de parcours parmi les possibilités offertes par le système. 


\section{Le roman comme fait cosmologique}

21 Cette conception combinatoire de l'imagination revient de façon puissante lorsqu'Eco explique sa technique pour la construction de ce qu'il appelle la «cosmologie» de ses romans; elle se base essentiellement sur la maîtrise parfaite des scripts, des scénarios narratifs d'une culture, d'une manière qui semble presque robotique dans son binarisme : qu'est-ce qui arrive à un pêcheur le long du rivage? Soit les poissons mordent à l'hameçon, soit ils n'y mordent pas. Eco nous fait croire que son écriture est une sorte de calcul, un choix entre les possibilités narratives du système, une espèce de pachinko textuel où, une fois choisi le premier point d'insertion de la bille, c'est le système ensuite qui en détermine le parcours. Tout ce qui, dans un roman, ne répond pas à ce mécanisme est, selon Eco, de la poésie, dans laquelle « verba tene, res sequentur ». Faut-il en déduire que la prose d'Eco, dans sa fidélité extrême aux lois de vraisemblance selon lesquelles se structurent les mondes possibles imaginés par une culture, est sans véritable invention qui ne soit pas combinatoire, et sans véritable "poésie », conçue comme la capacité non seulement de découvrir les possibilités du réel dans la parole, mais de les inventer?

"Il faut se créer des constructions pour pouvoir inventer librement", voici le mantra qu'Eco répète tout le temps dans « l'Apostille » aussi bien que dans ses écrits académiques sur la narration, adhérant ainsi à une conception quelque peu régimentaire de l'imagination. Pourquoi ces constructions? Pour attribuer la dignité de la vraisemblance à un roman historique? Mais qu'importe de construire ce cosmos parfait, ce jouet en tout pareil aux maisons de poupées les plus sophistiquées, s'il n'est pas habité par la vraisemblance qui intéresse le plus l'humanité, celle des émotions, celle que l'on retrouve dans les scénographies vides ou presque de la tragédie grecque, ou même dans les scénographies détaillées jusqu'à l'étouffement des grands romans russes, où pourtant l'on se retrouve non seulement au milieu d'un monde possible parfaitement meublé mais aussi au milieu d'un cœur possible parfaitement saignant?

Il ne faut pas toujours croire à « l'Apostille ». Par exemple, lorsqu'Eco nous raconte que, dans Le nom de la rose, il situe l'action à la fin de novembre car il avait besoin de tuer un cochon, et les cochons se tuent (« je me suis renseigné », dit-il) avec le froid. Cela n'a, en effet, aucune importance pour le lecteur. La plupart des lecteurs d'Eco n'ont peut-être même pas vu un cochon de leur vie, figurons-nous s'ils s'étonneraient en constatant que, dans le roman, l'auteur fait tuer un cochon en octobre (qui est assez froid, d'ailleurs, dans plusieurs régions d'Europe). La nécessité de motiver ces détails narratifs a posteriori réside ailleurs : il faut alimenter le mythe de l'écrivain architecte d'un monde possible, mais il faut surtout transmettre l'idée que ce monde n'est pas inventé mais trouvé, repéré dans la culture plutôt que créé ; pourquoi ? Parce que cela aussi contribue à la fois à éloigner et à rendre plus mystérieuse la personnalité de l'auteur, lequel avoue avoir écrit ce qu'il a écrit par nécessité, et non par désir.

Après tout, cette occultation des traces de la subjectivité de l'auteur, cet appel insistant à la nécessité que les mondes possibles narratifs suivent les constrictions dictées par la mémoire culturelle n'est qu'un exercice de motivation: je n'ai pas écrit un roman; le roman s'est fait écrire par moi. D'une part, cette attitude déresponsabilise l'écrivain par rapport à ses choix : ce qu'il a écrit, il l'a écrit pour obéir aux lois de l'invention narrative (à partir d'un coup d'envoi initial dont on se garde bien d'expliquer la nature); d'autre part, cette attitude élève le statut de l'écrivain du rang de créateur subjectif à celui de 
dépositaire d'une connexion mystérieuse aux "lois naturelles de l'imagination». Au bénéfice de cette rhétorique, Eco annonce, toujours dans "l'Apostille ", que "lorsque j'ai mis Jorge dans la bibliothèque je ne savais pas encore qu'il était l'assassin. Pour ainsi dire, il a tout fait à lui seul ». Voici l'un des mythes les plus persistants, presque un stéréotype, de la légende de l'écrivain: comme les légendes d'artistes nous le racontent, comme Michel-Ange affirmant non pas d'inventer des formes mais de les libérer de la matière, les légendes que les écrivains racontent sur eux-mêmes affirment la liberté des personnages, l'indépendance de leurs mouvements et décisions et le fait que, après la mise en place d'une charpente initiale, le roman «s'écrit tout seul».

\section{Qui parle?}

C'est évidemment une illusion, voire une rhétorique. Aucune forme ne se cache dans le marbre, tout comme aucun personnage de roman ne dit ou fait rien sans que ses mots et ses actions ne soient déterminés par les décisions plus ou moins conscientes de l'auteur. Pourquoi alors cette nécessité de la nécessité, cette dénégation du rôle de l'auteur empirique, de sa vie, de ses goûts, de ses dégoûts, de son caractère, dans la mise en œuvre du roman? Eco avoue même avoir éliminé une page de son roman où Guillaume de Baskerville priait dans un moment d'émotion, car cette page émouvait Eco lui-même et car elle ne s'harmonisait pas avec le caractère du personnage. Faut-il peut-être conclure que les romans d'Eco sont cérébraux, froids, inaffectifs, une sorte de machine à calculer narrative où l'auteur à la fois exalte son rôle démiurgique et cache son humanité, en s'y soustrayant en tant que voix mais surtout en tant qu'auteur empirique, en tant que sujet avec une histoire et une tragédie, comme tous les êtres humains? Raconter, affirme Eco, c'est « penser avec les doigts » : l'idée d'un texte qui se fait en partie tout seul, en suivant le rythme que lui a attribué la première impulsion de l'écrivain, devient puissante. Et ce qui en résulte, en même temps, c'est l'idée d'un espace de séparation entre deux étapes de la narration, l'une, lorsque l'écrivain Eco décide de raconter la seule expérience sexuelle d'Adso par le bais de textes religieux mystiques et spirituels utilisant l'étreinte érotique comme métaphore; l'autre, lorsque l'auteur, derechef, disparait derrière ce dispositif narratif et en suit le rythme sans engagement personnel. "Aucune émotion ", écrit Eco, lorsqu'il s'agissait de raconter l'histoire d'Adso qui fait l'amour dans les cuisines du monastère, «comme si j'avais décidé de raconter une histoire d'amour en jouant $\mathrm{du}$ tambour ».

\section{Conclusion : une rose sans épines}

C'est dit explicitement dans « l'Apostille »: Eco prend du plaisir à interposer des couches, des écrins comme il les appelle, entre son propre sujet d'auteur empirique et la voix que les lecteurs peuvent retrouver dans Le nom de la rose, distillée à travers une articulation de plusieurs niveaux (Eco qui énonce Mabillon qui énonce Vallet qui énonce Adso qui énonce Guillaume). Peut-on y voir une manœuvre pour la «mort de l'auteur", proclamée et programmée par une grande partie des avant-gardes du vingtième siècle ? L'insertion de tous ces filtres énonciatifs dans la prose du roman se déploie en synergie avec l'agencement méticuleux d'un espace et d'un temps du récit: dans les trois directions (acteurs, lieu, période) l'auteur s'y montre obligé de respecter une structure que luimême s'est donnée, ou du moins qu'il a choisi d'adopter à partir des formules déposées 
dans la culture et ses genres discursifs. L'effet psychologique qui en résulte est, cependant, non pas d'impuissance mais, au contraire, un effet démiurgique : l'auteur empirique, caché derrière sa structure, donne le coup d'envoi à la grande machine de la narration, laquelle, ensuite, se met à rouler toute seule, dans un mécanisme qui relève de la dimension de la fatalité.

Chez Eco, toutefois, l'ambition du démiurge, évidente dans toute sa production, s'adoucit par une fonction essentiellement protectrice: le texte, conçu comme machine combinatoire, doit surtout protéger l'auteur empirique de lui-même, constituer la gaine lui permettant de "s'échapper de l'émotion personnelle », une fuite qui est la façon dont Eco, d'après Joyce et Eliot, conçoit l'art. Dans l'une des rarissimes confessions personnelles de «l'Apostille », le grand savant italien l'affirme clairement: Adso, avec ses «trembleurs d'adolescent », c'est lui ; son histoire est l'histoire de quelqu'un qui ne vit la vie que par le truchement de la culture ; c'est l'histoire d'Eco. Mais n'est-ce pas là, peutêtre, l'enseignement moral le plus élevé et durable du Nom de la rose, à savoir la conviction douloureuse mais nécessaire que la seule façon de tolérer la vie et ses inquiétudes c'est de la transformer dans un monde possible autre, où elle se dérobe du chaos qui la marque et se case dans une structure ordonnée de lieux, de temps, d'acteurs et d'actions? N'est-ce pas cela que Le nom de la rose nous offre, au final, le sentiment que la seule rose sans épines est exactement celle que l'on transfigure dans un nom, dans le seul jardin que nous puissions vraiment habiter, le jardin du langage?

\section{BIBLIOGRAPHY}

Eco, Umberto. 1980. Il nome della rosa. Milan : Bompiani

Eco, Umberto. 1983. « Postille » a Il nome della rosa. Milan : Bompiani

\section{ABSTRACTS}

The semiotics of Umberto Eco is prolonged by other means in his novels : much has been written on the subject. But the reverse is also true: Eco's novels continue in sibylline ways throughout his philosophical work. The great Italian scholar's prodigious narrative ability manifests itself well before the Name of the Rose, and applies principally to ideas and knowledge. In Eco, the novel dresses with actors, figures, and icons, as well as with a brilliant arrangement of places, times, and aspects, a very solid narrative structure, inheritor to the great popular narratives of the nineteenth century. The paper expounds on the rhetorical effects of this synergy between theorization and narration and focuses, in particular, on the configuration of subjectivity in Eco's works.

La sémiotique d'Umberto Eco n'est par confinée dans le périmètre de la recherche et de l'écriture académiques mais s'étale également dans ses romans. Vice versa, la verve narrative du grand savant italien n'est par bornée par les frontières de la fiction mais s'insinue élégamment dans la rhétorique de l'essai : Eco savait comment raconter le récit de ses idées. L'article questionne le 
croisement de théorisation philosophique et narration romanesque dans l'œuvre d'Umberto Eco, en se penchant, en particulier, sur le statut de la subjectivité dans l'une et dans l'autre : tandis que le savant Eco se montre souvent dans l'épopée de son travail intellectuel, l'écrivain de romans Eco se cache, au contraire, derrière un auteur modèle qui semble bâti en suivant les théories mêmes de la sémiotique interprétative d'Eco.

INDEX

Mots-clés: Eco, narration, théorisation, subjectivité, savoir narré

Keywords: Eco, theorization, narration, subjectivity, narrated knowledge

\section{AUTHOR}

MASSIMO LEONE

Université de Turin 